This is the author's version of the paper.

The final publication has appeared in Int. J. Number Theory 6 (2010), 1113-1116.

\title{
A note on smooth numbers in short intervals
}

\author{
Kaisa Matomäki * \\ Department of Mathematics, 20014 University of Turku, Finland
}

\begin{abstract}
We prove that, for any $\epsilon>0$, there exist a constant $C>0$ such that the interval $[x, x+C \sqrt{x}]$ contains numbers whose all prime factors are smaller than $x^{1 /(5 \sqrt{e})+\epsilon}$.
\end{abstract}

\section{Introduction}

Let $\psi(\mathcal{A}, y)$ denote the number of $y$-smooth integers in the set $\mathcal{A} \subset[x, 2 x]$. With $y$-smooth integer we mean an integer whose all prime factors are at most $y$. For a typical set $\mathcal{A}$, one would expect that

$$
\psi(\mathcal{A}, y) \approx \frac{|\mathcal{A}|}{x} \psi([x, 2 x], y)
$$

Besides being of theoretical interest, smooth numbers play a prominent role in computational number theory. For such applications, see for instance the recent survey [5]. The interval $\mathcal{A}=[x, x+\sqrt{x}]$ arises in many cases. Harman [6] has shown that

$$
\psi([x, x+\sqrt{x}], y) \gg x^{1 / 2}
$$

for $y=x^{1 /(4 \sqrt{e})}$. He also gave a result for slightly shorter intervals.

When the length of the interval is greater than $\sqrt{x}$, powerful Dirichlet polynomial methods can be applied. Friedlander and Granville [4] used them to show that (1) holds for $\mathcal{A}=[x, x+z]$ with

$$
\exp \left((\log x)^{5 / 6+o(1)}\right) \leq y \leq x \quad \text { and } \quad \sqrt{x} y^{2} \exp \left((\log x)^{1 / 6}\right) \leq z \leq x .
$$

For intervals of length $\ll \sqrt{x}$ such small values of $y$ seem to be a distant target. However some progress has been made: Recently Croot [2] proved that there exists a constant $C=C(\epsilon)$ such that

$$
\psi\left([x, x+C \sqrt{x}], x^{47 /(190 \sqrt{e})+\epsilon}\right)>\sqrt{x}(\log x)^{-\log 4-o(1)}
$$

*Email address: ksmato@utu.fi

Keywords: Smooth numbers, short intervals

MSC (2000): 11N25 
for all sufficiently large $x$. Notice that $47 / 190 \approx 0.247$, so this slightly improves the smoothness parameter $y$ in Harman's result at the cost of a bit longer interval and a lower bound which is not of the expected order. In this paper we show how Croot's new approach can be refined to get the following result.

Theorem 1.1. Let $\epsilon>0$. There exists a constant $C=C(\epsilon)$ such that

$$
\psi\left([x, x+C \sqrt{x}], x^{1 /(5 \sqrt{e})+\epsilon}\right)>\sqrt{x}(\log x)^{-\log 4-o(1)}
$$

for all sufficiently large $x$.

The improvement comes from taking advantage of a result on sums over arithmetic progressions in [1].

\section{Proof of Theorem 1.1}

First we introduce some notation. Let $\delta$ be a small positive constant,

$$
\begin{aligned}
& Z=\left[(1+\delta)^{-1} \sqrt{x},(1+\delta) \sqrt{x}\right] \cap \mathbb{Z}, \\
& D=\left\{p_{1} p_{2} p_{3} \mid p_{i} \in\left[x^{1 / 10-\epsilon / 5}, x^{1 / 10-\epsilon / 10}\right]\right\}
\end{aligned}
$$

and

$$
h(n)=|\{q \in D|q| n\}| .
$$

The expected value of $h(n)$ over $n \in Z$ is

$$
\mathbb{E}(h)=\frac{1}{|Z|} \sum_{n \in Z} h(n)=\frac{1}{|Z|} \sum_{q \in D}\left(\frac{|Z|}{q}+O(1)\right)=\sum_{q \in D} \frac{1}{q}+O\left(\frac{|D|}{|Z|}\right) \gg \epsilon^{3} .
$$

We will show that $h$ behaves in expected manner in almost all very short intervals.

Lemma 2.1. For $\delta \in\left(0, \delta_{0}(\epsilon)\right)$ and $k>k_{0}(\epsilon, \delta)$, we have

$$
V=\sum_{z \in Z}\left(\sum_{n \in[z, z+k]} h(n)-(k+1) \mathbb{E}(h)\right)^{2} \leq \delta^{2} \mathbb{E}(h)^{2}(k+1)^{2}|Z|
$$

for all sufficiently large $x$.

This means that for most $z \in Z$, the interval $[z, z+k]$ contains about expected number of integers that are divisible by a member of $D$. These are automatically $x^{1 / 2-3 / 10+3 \epsilon / 5}=x^{1 / 5+3 \epsilon / 5}$-smooth. However, in considerations of smooth numbers it is often possible to reduce the smoothness parameter by the factor $1 / \sqrt{e}$, which is the case also here. Indeed, by the method of $[2$, Section 2.4], Lemma 2.1 implies that at least $\left(\delta+\delta^{2}\right) \sqrt{x}$ of the integers $z \in Z$ satisfy

$$
\psi\left([z, z+k], x^{1 /(5 \sqrt{e})+\epsilon}\right)>0 .
$$


Now we know that a bit more than a half of the integers $z \in Z$ satisfy (2). As shown in $\left[2\right.$, Section 2.2], this immediately implies that there are $\gg x^{1 / 2}$ pairs $z_{1}, z_{2}$ satisfying $(2)$ such that $z_{2}=\left\lceil x / z_{1}\right\rceil$. Then $\left(z_{1}+j_{1}\right)\left(z_{2}+j_{2}\right) \in \mathcal{A}$ and is $y$-smooth for some $j_{1}, j_{2} \in\{1,2, \ldots, k\}$. As in [2, Section 2.1], this implies Theorem 1.1. Hence we only need to prove Lemma 2.1.

Proof of Lemma 2.1. Squaring out, we see that

$$
\begin{aligned}
V & =\sum_{z \in Z}\left(\sum_{n \in[z, z+k]} h(n)\right)^{2}-2(k+1) \mathbb{E}(h) \sum_{z \in Z} \sum_{n \in[z, z+k]} h(n)+(k+1)^{2} \mathbb{E}(h)^{2}|Z| \\
& =\sum_{z \in Z}\left(\sum_{n \in[z, z+k]} \sum_{\substack{q \in D \\
q \mid n}} 1\right)^{2}-(k+1)^{2} \mathbb{E}(h)^{2}|Z|+O(k) \\
& =2 \sum_{j=1}^{k}(k+1-j) \sum_{q, q^{\prime} \in D} \sum_{\substack{n \in Z \\
q\left|n, q^{\prime}\right| n+j}} 1-(k+1)^{2} \mathbb{E}(h)^{2}|Z|+O(k|Z|) .
\end{aligned}
$$

Hence we need to consider the sum

$$
\sum_{q \in D} \sum_{q^{\prime} \in D} \sum_{\substack{d q \in Z \\ d q \equiv-j\left(\bmod q^{\prime}\right)}} 1 .
$$

The dependence $d q \in Z$ between $d$ and $q$ can be removed by splitting summations to short ranges as in [2, Section 2.5].

At this stage Croot handles the congruence condition using a finite Fourier transform and then applying the bound [3, Theorem 2] on bilinear forms with Kloosterman fractions.

However, sums of the type (3) have been studied in a greater depth in a series of papers by Bombieri, Friedlander and Iwaniec. In our situation [1, Theorem $5]$ is applicable. Letting $q^{\prime}=p_{1}^{\prime} p_{2}^{\prime} p_{3}^{\prime}$, we can take $n=q, r=p_{1}^{\prime}$ and $q=p_{2}^{\prime} p_{3}^{\prime}$ in that theorem. Then

$$
\begin{aligned}
\sum_{q \in D} \sum_{q^{\prime} \in D} \sum_{\substack{d q \in Z \\
d q \equiv-j\left(\bmod q^{\prime}\right)}} 1 & =\sum_{q \in D} \sum_{q^{\prime} \in D} \frac{1}{\phi(q)} \sum_{\substack{d q \in Z \\
\left(d q, q^{\prime}\right)=1}} 1+O\left(|Z|(\log x)^{-A}\right) \\
& =\left(1+o_{\epsilon, \delta}(1)\right) \mathbb{E}(h)^{2}|Z| .
\end{aligned}
$$

Hence

$$
V=o_{\epsilon, \delta}\left((k+1)^{2} \mathbb{E}(h)^{2}|Z|\right)+O(k|Z|) .
$$

This finishes the proof of Lemma 2.1 and therefore that of Theorem 1.1.

\section{Acknowledgments}

The author was supported by the Finnish Cultural Foundation. 


\section{References}

[1] E. Bombieri, J. B. Friedlander, and H. Iwaniec, Primes in arithmetic progressions to large moduli, Acta Math., 156 (1986) 203-251.

[2] E. Croot, Smooth numbers in short intervals, Int. J. Number Theory, 3 (2007) 159-169.

[3] W. Duke, J. Friedlander, and H. Iwaniec, Bilinear forms with Kloosterman fractions, Invent. Math., 128 (1997) 23-43,

[4] J. B. Friedlander and A. Granville, Smoothing "smooth" numbers, Philos. Trans. Roy. Soc. London Ser. A, 345(1676) (1993) 339-347.

[5] A. Granville, Smooth numbers: computational number theory and beyond, in Algorithmic Number Theory: Lattices, Number Fields, Curves and Cryptography, ed. J. P. Buhler and P. Stevenhagen, MSRI Publications, Vol. 44 (Cambridge University Press, Cambridge, 2008), pp. 267-323.

[6] G. Harman, Integers without large prime factors in short intervals and arithmetic progressions, Acta Arith., 91 (1999) 279-289. 